\title{
RKKY interactions in the regime of strong localization
}

\author{
J. A. Sobota, ${ }^{1}$ D. Tanasković, ${ }^{2,3}$ and V. Dobrosavljević ${ }^{3}$ \\ ${ }^{1}$ School of Applied and Engineering Physics, Cornell University, Ithaca, NY 14853, USA. \\ ${ }^{2}$ Institute of Physics, P.O. Box 57, 11080 Belgrade, Serbia. \\ ${ }^{3}$ Department of Physics and National High Magnetic Field Laboratory, \\ Florida State University, Tallahassee, Florida 32306, USA.
}

\begin{abstract}
We study the influence of strong nonmagnetic disorder on the Ruderman-Kittel-Kasuya-Yosida (RKKY) interactions between diluted magnetic moments in metals. We find that the probability distribution for the RKKY interactions assumes strongly non-Gaussian form featuring long tails. Since such distributions cannot be characterized by its moments, we define a typical value of the interaction amplitude, which we find to be exponentially suppressed in presence of Anderson localization. Our results present a plausible and physically transparent picture describing how Anderson localization effectively eliminates the long range nature of the RKKY interactions.
\end{abstract}

PACS numbers: $71.30 .+\mathrm{h}, 72.15 . \mathrm{Rn}, 73.20 . \mathrm{Fz}$

\section{INTRODUCTION}

It has long been appreciated that localized magnetic moments in metals interact through indirect RKKY interactions mediated by the conduction electrons $\stackrel{1}{=}$ In a clean metal, the RKKY interaction has a long-range oscillatory part, with an amplitude which decreases as a power law of the distance between the impurities, $I(R) \sim \cos \left(2 k_{F} R\right) / R^{d}$ ( $d$ is the dimensionality of the system and $k_{F}$ the Fermi wave vector). This behavior is well understood to be a direct consequence of the existence of a sharp Fermi surface characterizing itinerant electrons.

In presence of impurities and disorder, this behavior may be substantially modified. It is well known that sufficiently strong disorder can lead to multiple-scattering processes which can trap the electrons through the processes of Anderson localization. In this regime, one may expect that the long-range character of RKKY interactions should be suppressed, reflecting the reduced mobility of conduction electrons. The essential physical question is how this process precisely takes place as the disorder strength is gradually increased and the system approaches the localized regime.

The influence of weak nonmagnetic disorder on the RKKY interactions has been studied in considerable detail, $, 2,3,4,5,6$ and is by now very well understood. These studies have established that the main effect of weak disorder is to randomly modify the phase of the RKKY oscillations due to impurity-induced phase shifts of the electronic wave functions. As a result, the RKKY interaction decreases exponentially when averaged over disorder, $\langle I(R)\rangle \sim e^{-R / l}$, where $l$ is the mean free path. Early work ${ }^{3}$ thus predicted that the range of RKKY interactions becomes essentially cut off at $R \sim l$, and can be neglected at larger distances. More careful consideration ${ }^{4,5.6}$ discovered that this naive argument is incorrect, essentially because most relevant quantities do not depend on the average value of the interaction, but instead on the typical value of its amplitude. Since this quantity is not affected by the random phase shifts, it is essentially unaffected by weak disorder, and decreases with the distance $R$ in the same power-law fashion as in the clean system. Therefore, the presence of weak disorder is not expected to lead to any significant changes in the physical properties which are dominated by the RKKY interactions.

The influence of stronger nonmagnetic disorder on RKKY interactions has been, however, so far poorly explored. In an important study, Lerner examined the probability distribution of the RKKY interactions in the metallic phase in the presence of strong disorder within generalized nonlinear $\sigma$ model and $2+\varepsilon$ expansion ${ }^{\underline{7}}$ It was found that the quantum interference corrections do not change the power-law decay of all the even moments of the interaction distribution, which remains the same as in the pure metal, but make the coefficients attached to these moments increase critically with disorder. As the Anderson localization regime is approached, the higher moments increase much faster than the variance, which therefore no longer represents a good characterization of the typical interaction strength. Although this work clearly points out to the importance of strong fluctuations of the RKKY interactions, it does not explain how does the distribution function evolve as one enters the regime of Anderson localization.

Within an Anderson insulator the electrons are bound to impurities, and thus can hardly be expected to generate the long-range part of the RKKY interaction. How can we have at the same time large moments of the distribution of RKKY interactions, along with its fast decay with the distance? At first glance these two arguments seem inconsistent and the situation confusing and paradoxical. Clarifying these issues is an interesting and important problem, since one expects the magnetic correlations to play a crucial role in the physics of disorderdriven metal-insulator transitions in general.

The resolution of this puzzle is in fact quite simple, as we explain in this paper. We find that in presence of strong disorder and localization, the distribution function develops a strongly non-Gaussian form featuring 
long tails. In such cases it is well known that all moments of the distribution can assume very large values, while at the same time a typical width of the distribution can remain very small. Instead of the arithmetic average (i.e. standard deviation of the distribution) we find that the typical value of the interaction is better characterized by the geometric average of the distribution, $I_{t y p}(R) \equiv e^{\left\langle\frac{1}{2} \ln [I(R)]^{2}\right\rangle}$. This quantity is exponentially suppressed in the presence of Anderson localization, explaining how the long-range part of the RKKY interaction is suppressed.

To illustrate these ideas and obtain quantitative and reliable results, we numerically study the distribution of the RKKY interactions within the Anderson insulator phase. Although our numerical results are obtained within one dimensional model, we argue that the same concept of the typical value of the RKKY interaction can be used to physically explain the qualitative change of the form of RKKY interactions from long ranged to short ranged during the disorder driven metal-insulator transition in general dimensions. In the rest of this paper we present a detailed description of the model, followed by the numerical results and discussion.

\section{RKKY INTERACTIONS IN A DISORDERED METAL}

The interaction energy between two local moments $\mathbf{S}_{1}$ and $\mathbf{S}_{2}$ embedded in the metallic host at $\mathbf{r}_{1}$ and $\mathbf{r}_{2}$, respectively, is given by the Hamiltonian

$$
H_{i n t}=-J^{2} \mathbf{S}_{1} \cdot \mathbf{S}_{2} \chi\left(\mathbf{r}_{1}, \mathbf{r}_{2}\right),
$$

where $\chi$ is the zero frequency nonlocal electronic susceptibility and $J$ is the exchange coupling constant. Therefore the calculation of the interaction between diluted magnetic impurities reduces to the calculation of the susceptibility which, expressed through the Matsubara Green's functions, is given by

$$
\chi\left(\mathbf{r}_{1}, \mathbf{r}_{2}\right)=\frac{2}{\beta} \sum_{\omega_{n}} G_{\omega_{n}}\left(\mathbf{r}_{1}, \mathbf{r}_{2}\right) G_{\omega_{n}}\left(\mathbf{r}_{2}, \mathbf{r}_{1}\right) .
$$

Here $\omega_{n}$ is the fermionic Matsubara frequency, and $\beta$ is the inverse temperature.

In the clean case, i.e. in the absence of the nonmagnetic disorder, in three dimensional (3d) system, and for $R$ much larger than the lattice spacing, the susceptibility is equal to

$$
\chi_{o}(R)=-\frac{2 m k_{F} \cos \left(2 k_{F} R\right)}{(2 \pi)^{3} R^{3}},
$$

where $R=\left|\mathbf{r}_{1}-\mathbf{r}_{2}\right|$ and $m$ is the effective mass.

In the presence of weak disorder the phase of $\chi(R)$ becomes random and $\chi(R)$ averaged over the disorder configurations is exponentially suppressed

$$
\langle\chi(R)\rangle=\chi_{o}(R) e^{-R / l},
$$

where $l$ is the mean free path. $2,3,4,5,6$ The second moment (variance) of the probability distribution, however, remains long ranged and has the same power law dependence as in the clean system $4,5,6$

$$
\left\langle\chi^{2}(R)\right\rangle=3\left[\frac{m k_{F}}{(2 \pi)^{3}}\right]^{2} \frac{1}{R^{6}}
$$

Jagannathan et al. $\frac{6}{}$ have found that the square root of the forth moment of the distribution, $\sqrt{\left\langle\chi^{4}\right\rangle}$, is comparable in magnitude to the second moment. Therefore, the susceptibility distribution is non-Gaussian, but its typical value is well characterized by the square root of its second moment. The same remains true in $2 d$ as well $\underline{6}$

This statement is, however, not valid in the regime of strong disorder. As shown by Lerner, $\frac{7}{-}$ using the generalized nonlinear $\sigma$ model and performing a $2+\varepsilon$ expansion, further increase of the disorder results in very rapid increase of all the even cumulants of the distribution. More precisely, for the cumulant $\left\langle\left\langle\chi^{n}\right\rangle\right\rangle$ of the order $n$

$$
\frac{\left\langle\left\langle\chi^{n}(R)\right\rangle\right\rangle}{R^{-n d}} \sim e^{2 u n^{2}},
$$

where the parameter $u \gtrsim 1$ as the disorder is increased and the system approaches the Anderson transition. ${ }^{7}$ In this case the typical value of the distribution cannot be determined by the value of its moments.

\section{NUMERICAL RESULTS}

In order to examine the form of the RKKY interactions in the regime of strong disorder, we proceed to a numerical study. We consider a tight binding model

$$
H=-t \sum_{\langle i j\rangle \sigma}\left(c_{i \sigma}^{\dagger} c_{j \sigma}+\text { h.c. }\right)+\sum_{i \sigma} \varepsilon_{i} c_{i \sigma}^{\dagger} c_{i \sigma}
$$

with nearest neighbor hopping $t$ and on-site random potential $\varepsilon_{i}$, which is distributed uniformly in the interval $[-W / 2, W / 2]$. We calculate the interaction between the magnetic impurities embedded into this system at distance $R$. From Eqs. (2.1) and (2.2) we see that all the information that we need is contained in the single particle Green's functions $G_{\omega_{n}}\left(\mathbf{r}_{1}, \mathbf{r}_{2}\right)$. In the matrix notation the Green function

$$
\hat{G}\left(\omega_{n}\right)=\left(i \omega_{n}-\hat{H}\right)^{-1}
$$

and the problem reduces to the numerical summation of the corresponding matrix elements over Matsubara frequencies $\omega_{n}$ which will be done in the zero temperature limit. In order to obtain good statistics with large number of disorder realizations and to reduce the finite size effects, we concentrate in the following to a one dimensional system.

The numerical results for the clean system, Fig. 1, reproduce the well known oscillatory form of the electronic 


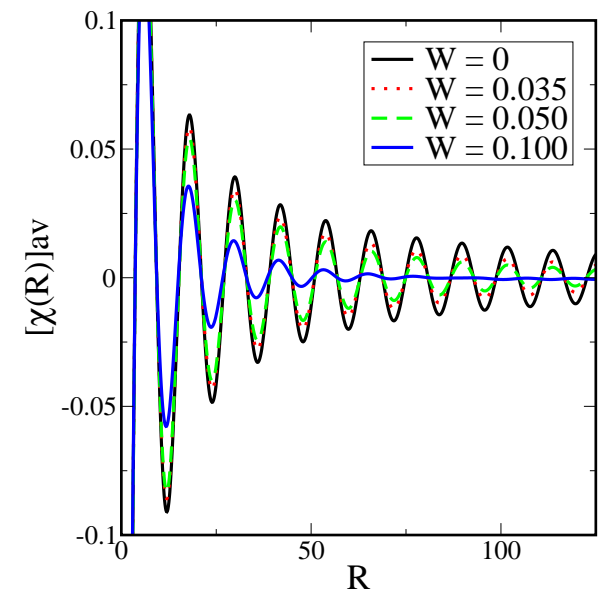

Figure 1: The average susceptibility as a function of distance $R$ in the presence of weak disorder of strength $W$. The chemical potential is $\mu=-0.96$.

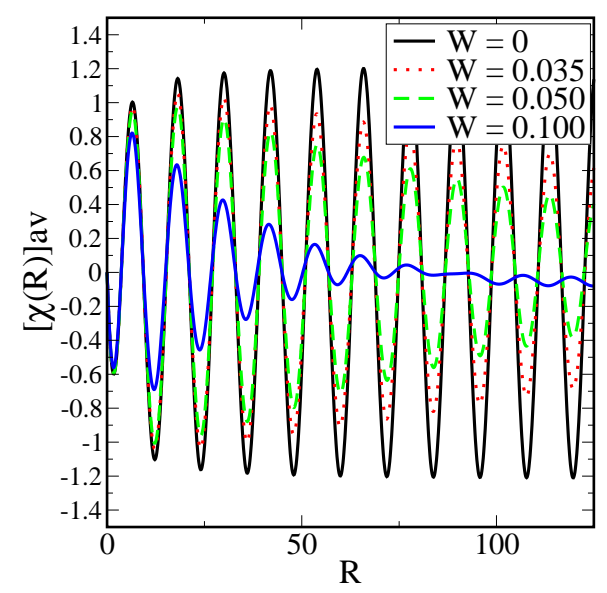

Figure 2: Scaled average susceptibility $R\langle\chi(R)\rangle$. With the $1 / R$ dependence gone, we can see that disorder introduces a damping factor to the average interaction strength.

susceptibility with the power law decay with the distance, $\chi(R) \sim \cos \left(2 k_{F} R\right) / R$. Here the power law exponent is equal to 1 since we are working within one dimensional (1d) model. (The disorder strength $W$ and the chemical potential $\mu$ are measured in units of half the bandwidth $2 t$, and the distance $R$ in units of the lattice spacing. Our system had 500 lattice sites.) We then consider the susceptibility $\langle\chi(R)\rangle$ in presence of weak disorder averaged over hundreds of disorder configurations. The average susceptibility weakens as the disorder is increased.

In Fig. 2 we remove the $1 / R$ dependence by multiplying the average value $\langle\chi\rangle$ by $R$. For weak disorder $\langle\chi\rangle R$ follows an exponential decay as predicted long time ago by de Gennes. ${ }^{3}$ However, what we are really interested in are the probability distributions of the electronic susceptibility in the presence of stronger disorder.

Fig. 3 shows the probability distribution of the scaled

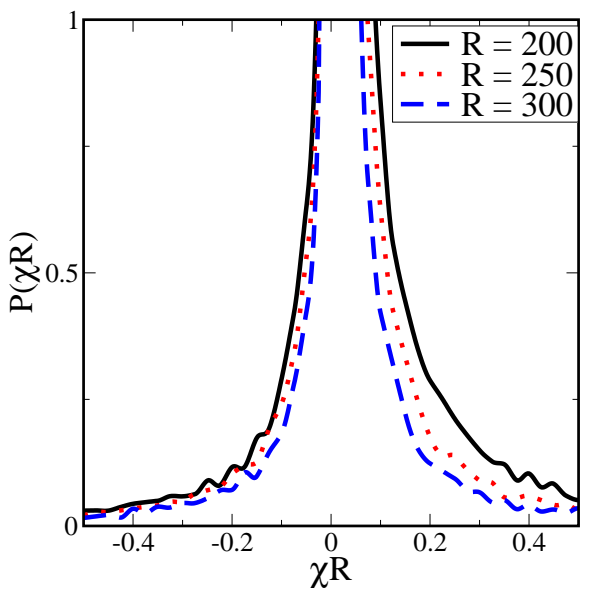

Figure 3: Probability distribution $P(\chi R)$ for $W=0.35$ and $\mu=-0.96$. The distribution width is clearly dependent on $R$.

susceptibility $P(\chi R)$ in the presence of strong disorder, and for several values of $R$. As in the remaining part of the paper, the results are obtained by averaging over hundreds of disorder configurations on the lattice with $500-1000$ lattice sites. The width of the distributions are dependent on $R$. More importantly, cursory examination of the data indicates that the distributions are distinctly non-Gaussian. This feature is important for the following analysis, since the non-Gaussian shape of the distribution prevents us from using the standard deviation as a measure of the the width of the distributions. Instead, we define a typical value of the width as the geometrical average

$$
\chi_{\text {typ }}(R) \equiv e^{\left\langle\frac{1}{2} \ln \chi^{2}(R)\right\rangle} .
$$

Fig. 4 shows a comparison of standard deviation and $\chi_{t y p}$ for describing the width of the same distribution. We can see that the standard deviation is influenced by the long tails, and is far too large to be a useful description of the width. $\chi_{t y p}$, however, gives a good estimate of the width of the distribution.

Examining $\chi_{t y p}$ for different strengths of disorder (Figs. 5 and 6), we find that for each value of the disorder $W$

$$
R \chi_{t y p}(R) \sim e^{-R / \xi}
$$

for sufficiently large $\mathrm{R}$, where $\xi$ defines the localization length. Therefore, if we define an adjusted susceptibility,

$$
\chi_{A}(R) \equiv R e^{R / \xi} \chi(R)
$$

the distributions of $\chi_{A}(R)$ for large $\mathrm{R}$ will be entirely independent of $\mathrm{R}$ within each disorder strength. Comparing Figs. 3 and 7 shows how the use of this adjusted susceptibility causes these distributions to collapse to a single scaling function. We then combine the data for 


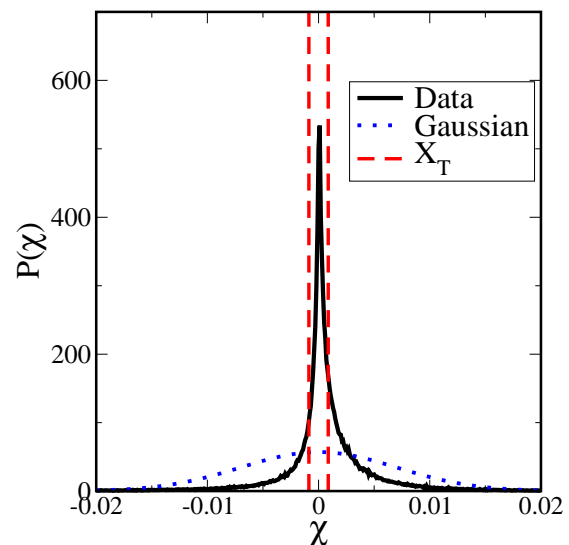

Figure 4: Distribution $P(\chi)$ (full black line) for $R=100$ and $W=0.2$. Gaussian (blue dotted line) is taken to have the same standard deviation as $P(\chi) . \chi_{t y p}$ (red dashed line) is a better measure of the distribution width than its standard deviation.

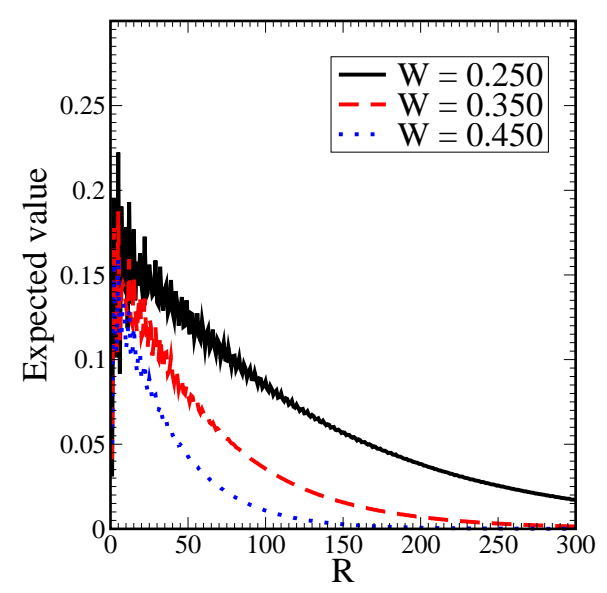

Figure 5: $R \chi_{\text {typ }}$ on linear axes. The width of the distributions decrease with increasing $R$.

several distances $R$, and thereby obtain more precise distributions for each value of disorder $W$. For each disorder strength there now is a single characteristic distribution (independent on $R$ ) as shown in Fig. 8. Interestingly, the probability distributions are quite asymmetric in the presence of stronger disorder, ferromagnetic interactions being much more probable than the antiferromagnetic ones. In the strongly localize regime we expect in fact the interactions to be ferromagnetic for $\mathrm{R}$ smaller or of the order of the localization length. The obtained distributions show this behavior even for $\mathrm{R}$ much larger that the localization length.

We then plot the tails of the distributions and find that for very strong disorder they become very long, as shown in Fig. 9. The tails of the distribution appear to converge to a universal power law form in the limit of strong disorder. The form of the tails is qualitatively the

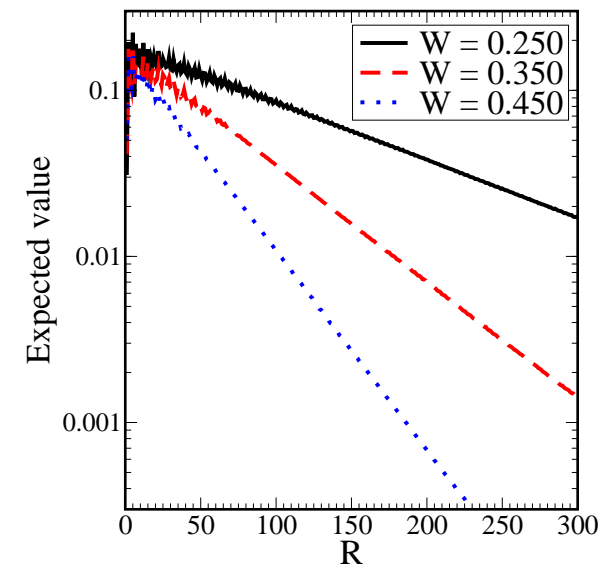

Figure 6: $R \chi_{t y p}$ on semilogarithmic axes. $R \chi_{t y p}$ features an exponential decay with $R$.

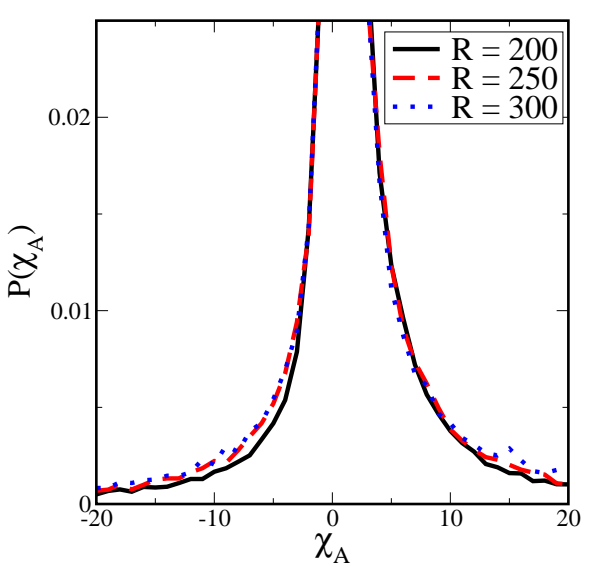

Figure 7: Scaled $P\left(\chi_{A}\right)$ for $\mathrm{W}=0.350$. After scaling, the distributions collapse.

same for positive and negative side of the distribution. The existence of such long tails indicates that the width of the distributions for higher disorder cannot be accurately characterized by their moments. The moments of such a distribution are extremely large, while the typical value is, in fact, very small. Therefore, the long range part of the RKKY interactions is strongly suppressed in the strongly localization regime.

We have also compared our result for the mean free path $l$, obtained from Eq. (2.4), and the localization length $\xi$, from Eq. (3.4). Plotting $l$ and $\xi$ as a function of $W$, see Fig. 10, we find that $l$ and $\xi$ are both proportional to $W^{-2}$, and find that $\xi / l \approx 3.685$, which is in a good agreement with the analytical result $\xi / l \approx 4.8$ This analysis further confirms the consistency of our interpretation of $\xi$, as determined from the decay of the typical RKKY interaction amplitude, with the localization length of the electronic system. 


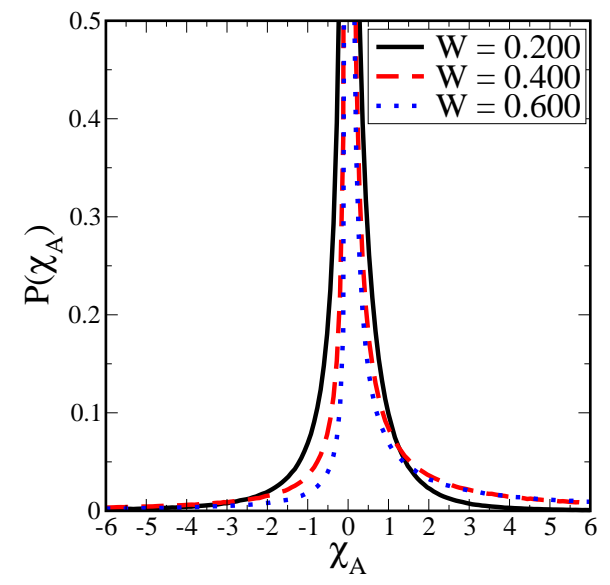

Figure 8: $P\left(\chi_{A}\right)$ for several values of $W$. The distributions are strongly peaked for higher disorder.

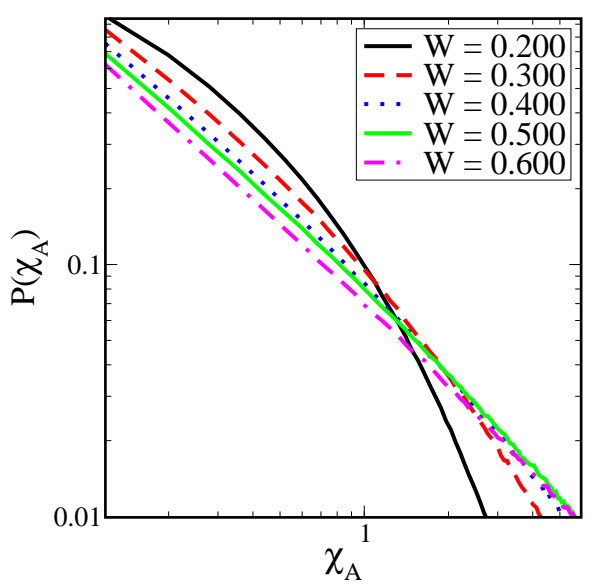

Figure 9: Tails of distributions on log-log axes. For higher disorder, the tails become increasingly long.

\section{CONCLUSION}

In this paper we have examined how the distribution function for RKKY interactions becomes modified due to Anderson localization effects. We demonstrated that the essential effect of localization is to exponentially suppress the typical amplitude of RKKY interactions on distances longer then the localization length, in agreement with intuitive expectations. The distribution, nevertheless, remains "broad" in the sense that it develops long tails which dominate the statistics. Our numerical results thus confirm the analytical predictions of Lerner that all even moments of the distribution diverge within an Anderson insulator.

Our results portray an interesting physical picture with potentially far-reaching consequences. In the metallic regime the RKKY interactions remain long-ranged even in presence of weak disorder, and thus a given magnetic moment effectively interacts with many others. In this

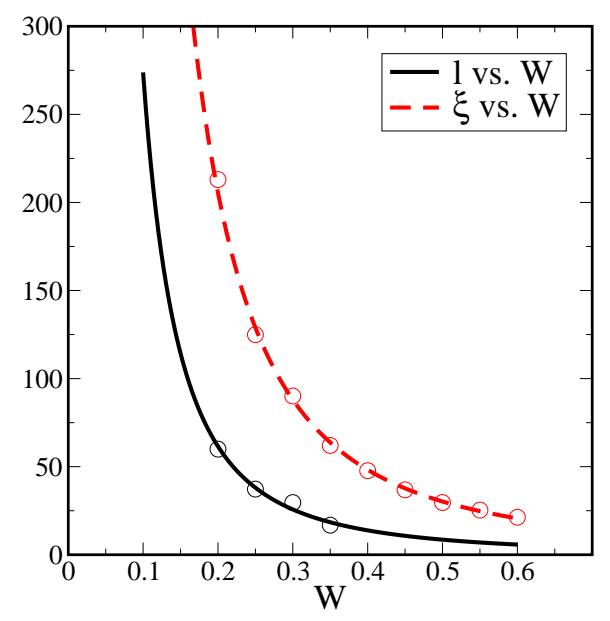

Figure 10: The mean free path $l$ and the localization length $\xi$ as a function of the disorder strength. Both $l$ and $\xi$ are found to be proportional to $W^{-2}$.

regime one may expect a well developed collective behavior of the spin system, leading to magnetic ordering at low temperature. When the Anderson-localized regime is approached, the situation is quite different. The RKKY interaction between a typical pair of distant spins is now significantly suppressed or even negligibly small. Very occasionally, a pair of distant spins will interact strongly, due to rare disorder configurations producing long tails in the distribution function. If the resulting RKKY interaction is antiferromagnetic, then such a pair can be expected to lock in a tightly bound singlet thus forming an essentially inert molecule that practically detaches from the rest of the spin system. Such process are precisely what one expects within the random singlet phases $910,11,12$ which feature quantum Grif-

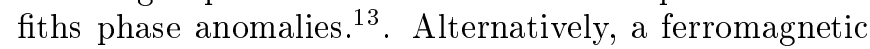
interaction will lead to the formation of a bound triplet state, essentially a spin $S=1$ magnetic moment. It is interesting to note a degree of asymmetry of the distribution of the RKKY interactions, that we have found at strong disorder. This finding seems to indicate that ferromagnetic correlations may effectively compete with the tendency for singlet formation, possibly leading to nanoscale ferromagnetism ${ }^{14}$ coexisting with a random singlet phase.

We thus anticipate that Anderson localization processes generically destabilize spin glass ordering in disordered metals, which is instead replaced by an appropriate quantum Griffiths phase. Precisely how these processes take place in the vicinity of realistic metal-insulator transitions remains a fascinating open direction for future study. 


\section{ACKNOWLEDGEMENTS}

We thank E. Abrahams, R. Bhatt, S. Chakravarty, E. Miranda, and B. Spivak for useful discussions. This work was supported by the National High Magnetic Field Lab- oratory, NSF grants DMR-0234215 (V.D. and D.T.) and DMR-0542026 (V.D.), Center for Integrating Research and Learning stuff (J.A.S.), and Serbian Ministry of Science, Project No. 141014 (D.T.).
1 M. A. Ruderman and C. Kittel, Phys. Rev. 96, 99 (1954); T. Kasuya, Phys. Rev. 106, 893 (1957); K. Yosida, Prog. Theor. Phys. 16, 45 (1956).

${ }^{2}$ P. F. de Chatel, Magn. Magn. Mater. 23, 28 (1981).

3 P. G. de Gennes, J. Phys. Radium 23, 630 (1962).

4 A. Yu. Zyuzin and B. Z. Spivak, Pis'ma Zh. Eksp. Teor. Fiz. 43, 185 (1986) [JETP Lett. 43, 234 (1986)].

${ }^{5}$ L. N. Bulaevskii and S. V. Panyukov, Pis'ma Zh. Eksp. Teor. Fiz. 43, 190 (1986) [JETP Lett. 43, 240 (1986)].

6 A. Jagannathan, E. Abrahams, and M. J. Stephen, Phys. Rev. B 37, 436 (1988).

7 I. V. Lerner, Phys. Rev. B 48, 9462 (1993).

8 E. N. Economou, Green's Functions in Quantum Physics, (Springer-Verlag, Berlin, 1983).

9 R. N. Bhatt and P. A. Lee, J. Appl. Phys. 52, 1703 (1981);
Phys. Rev. Lett. 48, 344 (1982).

10 C. Dasgupta and S. k. Ma, Phys. Rev. B 22, 1305 (1980); S. k. Ma, C. Dasgupta, and C. k. Hu, Phys. Rev. Lett. 43, 1434 (1979).

11 M. A. Paalanen and R. N. Bhatt, Physica B 169, 231 (1991).

12 M. P. Sarachik, in Metal-Insulator Transitions Revisited, edited by P. Edwards and C. N. R. Rao (Taylor and Francis, 1995).

13 E. Miranda and V. Dobrosavljević, Rep. Prog. Phys. 68, 2337 (2005).

14 E. Nielsen and R. N. Bhatt, preprint arXiv/cond-mat.strel/0705.2038. 